\title{
Existence and uniqueness of solution for Sturm-Liouville fractional differential equation with multi-point boundary condition via Caputo derivative
}

\author{
Ahmed M.A. El-Sayed ${ }^{1}$ and Fatma M. Gaafar ${ }^{2 *}$
}

"Correspondence:

fatmagaafar2@yahoo.com

${ }^{2}$ Department of Mathematics,

Faculty of Science, Damanhour

University, Damanhour, Egypt

Full list of author information is

available at the end of the article

\begin{abstract}
We investigate the existence and uniqueness of a solution for a Sturm-Liouville fractional differential equation with a multi-point boundary condition via the Caputo derivative; existence and uniqueness results for the given problem are obtained via the Banach fixed point theorem. Also we study its continuous dependence on coefficients of the nonlocal condition. We discuss our results for more general boundary conditions, we present the existence of solutions under nonlocal integral conditions and also extend our results to an ordinary Sturm-Liouville problem. Two examples illustrating the main results are also presented.
\end{abstract}

MSC: 26A33; 34B24; 34B10

Keywords: Fractional Sturm-Liouville problem; Caputo derivatives; Existence and uniqueness; Multi-point boundary condition; Continuous dependence;

Riemann-Stieltjes integral condition

\section{Introduction}

Consider the Sturm-Liouville fractional differential problem

$$
\begin{aligned}
& { }^{c} D^{\alpha}\left(p(t) u^{\prime}(t)\right)+q(t) u(t)=h(t) f(u(t)), \quad t \in(0, T), \\
& u^{\prime}(0)=0, \quad \sum_{k=1}^{m} \xi_{k} u\left(a_{k}\right)=v \sum_{j=1}^{n} \eta_{j} u\left(b_{j}\right),
\end{aligned}
$$

where $\alpha \in(0,1],{ }^{c} D^{\alpha}$ denotes the Caputo fractional derivative, $p(t) \in C^{1}(\mathcal{J}, \mathcal{R})$ and $q(t)$ and $h(t)$ are absolute continuous functions on $\mathcal{J}=[0, T], T<\infty$ with $p(t) \neq 0$ for all $t \in \mathcal{J}$, $f(u(t)): \mathcal{R} \rightarrow \mathcal{R}$ is defined and differentiable on the interval $\mathcal{J}, 0 \leq a_{1}<a_{2}<\cdots<a_{m}<$ $c, d \leq b_{1}<b_{2}<\cdots<b_{n} \leq T, c \leq d$ and $\xi_{k}, \eta_{j}$ and $v \in \mathcal{R}$. In this work, we discuss the existence and uniqueness of the solution $u(t) \in C^{1}(\mathcal{J}, \mathcal{R})$ of the Sturm-Liouville fractional differential equation (1.1) with the multi-point boundary condition (1.2). We also study the continuous dependence of the solution on the coefficients $\xi_{k}$ and $\eta_{j}$ of the multi-point boundary condition.

(c) The Author(s) 2019. This article is distributed under the terms of the Creative Commons Attribution 4.0 International License (http://creativecommons.org/licenses/by/4.0/), which permits unrestricted use, distribution, and reproduction in any medium, provided you give appropriate credit to the original author(s) and the source, provide a link to the Creative Commons license, and indicate if changes were made. 
As a consequence of our results, we find a unique solution for the ordinary SturmLiouville differential equation with the multi-point boundary condition

$$
\begin{aligned}
& \left(p(t) u^{\prime}(t)\right)^{\prime}+q(t) u(t)=h(t) f(u(t)), \quad t \in(0, T), \\
& u^{\prime}(0)=0, \quad \sum_{k=1}^{m} \xi_{k} u\left(a_{k}\right)=v \sum_{j=1}^{n} \eta_{j} u\left(b_{j}\right),
\end{aligned}
$$

As an extension of our problem, we deduce the presence of a unique solution for Eqs. (1.1) and (1.3) under the integral conditions

$$
u^{\prime}(0)=0, \quad \int_{a}^{c} u(\theta) d \varpi(\theta)=v \int_{d}^{e} u(\theta) d \vartheta(\theta)
$$

where $\varpi(\theta)$ and $\vartheta(\theta)$ are an increasing functions and the integrals are meant in the Riemann-Stieltjes sense for $0 \leq a<c \leq d<e \leq T$.

Sturm-Liouville operator is an important operator in physics, applied mathematics and other fields of engineering and science and has wide applications in quantum mechanics, classical mechanics and wave phenomena; see Joannopoulos [10] and Teschl [25] and the references therein. The existence of solutions and other properties for Sturm-Liouville boundary value problems have received considerable attention from many researchers during the last two decades; see for example Al-Mdallal [1], Bensidhoum and Dib [2], Erturk [7], Hassana [9], Klimek and Argawal [12], Li et al. [13], Lian and Ge [14], Liu et al. [17], Muensawat et al. [20], Xu and Abernathy [26], Yang [27] and the references therein.

Nonlocal and multi-point conditions can be more useful than the standard initial condition to describe some physical phenomena and have widely been studied by several researchers; see Cui and Zou [3], EL-Sayed and Bin-Taher [4, 5], El-Shahed and Nieto [6], Guo et al. [8], Karaaslan [11], Liu et al. [16], Liang et al. [15], Ma [18], Nyamoradi [21, 22], Nyamoradi et al. [23], Zhao and Ge [30], Zhang and Zhong [28], Zhanga and Liu [29], Zhong and Lin [31] and the references therein.

\section{Preliminaries and main results}

First of all, we introduce some notations and basic facts which are used throughout the paper.

Let $\|u\|=\max \{|u(t)|: t \in[0, T]\}$ is the norm in the space $C[0, T]$ and $\|u\|_{L_{1}}=\int_{0}^{T}|u(t)| d t$ is the norm in $L_{1}[0, T]$.

The Riemann-Liouville fractional integral of order $\alpha>0$ for the function $u(t) \in L_{1}[0, T]$ is known (see [19], [24]):

$$
I^{\alpha} u(t)=\int_{0}^{t} \frac{(t-s)^{\alpha-1}}{\Gamma(\alpha)} u(s) d s
$$

and the Caputo fractional derivative of order $n-1<\alpha \leq n$ for the function $u(t)$ is known:

$$
D^{\alpha} u(t)=I^{n-\alpha} \frac{d^{n}}{d t^{n}} u(t)=\int_{0}^{t} \frac{(t-s)^{n-\alpha-1}}{\Gamma(n-\alpha)} \frac{d^{n} u(s)}{d s^{n}} d s
$$

and we have for $\alpha, \beta \in \mathcal{R}^{+}$: 
$\left(r_{1}\right) I^{\alpha}: L_{1} \rightarrow L_{1}$ and $\lim _{\alpha \rightarrow 1} I^{\alpha} f(t)=I^{1} f(t)=\int_{0}^{t} f(s) d s$.

$\left(r_{2}\right) I^{\alpha} I^{\beta} f(t)=I^{\alpha+\beta} f(t)$.

$\left(r_{3}\right)$ If $f(t)$ is absolutely continuous on $\mathcal{J}$, then $\lim _{\alpha \rightarrow 1}{ }^{c} D^{\alpha} f(t)=D f(t)$ and

$$
D I^{\alpha} f(t)=\frac{t^{\alpha-1}}{\Gamma(\alpha)} f(0)+I^{\alpha} D f(t)
$$

$\left(r_{4}\right) I^{\alpha} t^{\gamma}=\frac{\Gamma(\gamma+1) t^{\alpha+\gamma}}{\Gamma(\alpha+\gamma+1)}, \gamma+1>0$.

Here we investigate the Sturm-Liouville fractional differential equation (1.1) with the multi-point boundary condition (1.2) under the following assumptions.

$\left(D_{1}\right)$ The function $f: \mathcal{R} \rightarrow \mathcal{R}$ is defined and differentiable on the interval $[0, T]$ and $\frac{\partial f}{\partial u}$ is bounded on $\mathcal{J}$ with $\left|\frac{\partial f}{\partial u}\right| \leq \mathcal{K}$.

$\left(D_{2}\right)$ The function $p(t) \in C^{1}(\mathcal{J}, \mathcal{R})$ with $p(t) \neq 0$ for all $t \in \mathcal{J}, \inf _{[0, T]}|p(t)|=p$ and $q(t)$ and $h(t)$ are absolute continuous functions on $\mathcal{J}$.

Lemma 2.1 Let the assumptions $\left(D_{1}\right)-\left(D_{2}\right)$ be satisfied. Then problem $(1.1)-(1.2)$ is equivalent to the integral equation

$$
\begin{aligned}
u(t)= & E \sum_{k=1}^{m} \xi_{k} \int_{0}^{a_{k}} \frac{1}{p(s)} I^{\alpha} q(s) u(s) d s-v E \sum_{j=1}^{n} \eta_{j} \int_{0}^{b_{j}} \frac{1}{p(s)} I^{\alpha} q(s) u(s) d s \\
& -E \sum_{k=1}^{m} \xi_{k} \int_{0}^{a_{k}} \frac{1}{p(s)} I^{\alpha} h(s) f(u(s)) d s+v E \sum_{j=1}^{n} \eta_{j} \int_{0}^{b_{j}} \frac{1}{p(s)} I^{\alpha} h(s) f(u(s)) d s \\
& -\int_{0}^{t} \frac{1}{p(s)} I^{\alpha} q(s) u(s) d s+\int_{0}^{t} \frac{1}{p(s)} I^{\alpha} h(s) f(u(s)) d s,
\end{aligned}
$$

with $u \in C^{1}(\mathcal{J}, \mathcal{R})$ and $u^{\prime \prime}(t) \in L_{1}(\mathcal{J}, \mathcal{R})$, where $E=\frac{1}{\sum_{k=1}^{m} \xi_{k}-\nu \sum_{j=1}^{n} \eta_{j}}$.

Proof For $t>0$, Eq. (1.1) can be written as

$$
I^{1-\alpha} \frac{d}{d t} p(t) u^{\prime}(t)=-q(t) u(t)+h(t) f(u(t)) .
$$

Operating by $I^{\alpha}$ on both sides, we obtain

$$
\begin{aligned}
& I^{1} \frac{d}{d t} p(t) u^{\prime}(t)=-I^{\alpha} q(t) u(t)+I^{\alpha} h(t) f(u(t)), \\
& p(t) u^{\prime}(t)-p(0) u^{\prime}(0)=-I^{\alpha} q(t) u(t)+I^{\alpha} h(t) f(u(t)) .
\end{aligned}
$$

From (1.2) we have

$$
\begin{aligned}
& u^{\prime}(t)=-\frac{1}{p(t)} I^{\alpha} q(t) u(t)+\frac{1}{p(t)} I^{\alpha} h(t) f(u(t)) \\
& u(t)-u(0)=-\int_{0}^{t} \frac{1}{p(s)} I^{\alpha} q(s) u(s) d s+\int_{0}^{t} \frac{1}{p(s)} I^{\alpha} h(s) f(u(s)) d s .
\end{aligned}
$$


For convenience, put $A(t)=\int_{0}^{t} \frac{1}{p(s)} I^{\alpha} q(s) u(s) d s$ and $B(t)=\int_{0}^{t} \frac{1}{p(s)} I^{\alpha} h(s) f(u(s)) d s$, we get

$$
\sum_{k=1}^{m} \xi_{k} u\left(a_{k}\right)-\sum_{k=1}^{m} \xi_{k} u(0)=-\sum_{k=1}^{m} \xi_{k} A\left(a_{k}\right)+\sum_{k=1}^{m} \xi_{k} B\left(a_{k}\right)
$$

and

$$
v \sum_{j=1}^{n} \eta_{j} u\left(b_{j}\right)-v \sum_{j=1}^{n} \eta_{j} u(0)=-v \sum_{j=1}^{n} \eta_{j} A\left(b_{j}\right)+v \sum_{j=1}^{n} \eta_{j} B\left(b_{j}\right) .
$$

On subtracting (2.3) from (2.4) and using $\sum_{k=1}^{m} \xi_{k} u\left(a_{k}\right)=v \sum_{j=1}^{n} \eta_{j} u\left(b_{j}\right)$, we obtain

$$
u(0)=E\left[\sum_{k=1}^{m} \xi_{k} A\left(a_{k}\right)-v \sum_{j=1}^{n} \eta_{j} A\left(b_{j}\right)-\sum_{k=1}^{m} \xi_{k} B\left(a_{k}\right)+v \sum_{j=1}^{n} \eta_{j} B\left(b_{j}\right)\right],
$$

with $E=\frac{1}{\sum_{k=1}^{m} \xi_{k}-v \sum_{j=1}^{n} \eta_{j}}$, substituting in (2.2) we get (2.1).

Conversely, to complete the equivalence between integral equation (2.1) and problem (1.1)-(1.2), we have from (2.1)

$$
\begin{aligned}
& u^{\prime}(t)=-\frac{1}{p(t)} I^{\alpha} q(t) u(t)+\frac{1}{p(t)} I^{\alpha} h(t) f(u(t)) \in C(\mathcal{J}, \mathcal{R}), \\
& \frac{d}{d t}\left(p(t) u^{\prime}(t)\right)=-\frac{d}{d t} I^{\alpha} q(t) u(t)+\frac{d}{d t} I^{\alpha} h(t) f(u(t)), \\
& I^{1-\alpha} \frac{d}{d t}\left(p(t) u^{\prime}(t)\right)=-I^{1-\alpha} \frac{d}{d t} I^{\alpha} q(t) u(t)+I^{1-\alpha} \frac{d}{d t} I^{\alpha} h(t) f(u(t)) .
\end{aligned}
$$

From the definition of Caputo derivative and applying $\left(r_{3}\right)$, we have

$$
\begin{aligned}
{ }^{c} D^{\alpha}\left(p(t) u^{\prime}(t)\right)= & -I^{1-\alpha} I^{\alpha} \frac{d}{d t}(q(t) u(t))+I^{1-\alpha} I^{\alpha} \frac{d}{d t}(h(t) f(u(t))) \\
& -I^{1-\alpha} \frac{t^{\alpha-1}}{\Gamma(\alpha)} q(0) u(0)+I^{1-\alpha} \frac{t^{\alpha-1}}{\Gamma(\alpha)} h(0) f(u(0)) .
\end{aligned}
$$

Hence, from $\left(r_{2}\right)$ and $\left(r_{4}\right)$ we get

$$
\begin{aligned}
{ }^{c} D^{\alpha}\left(p(t) u^{\prime}(t)\right) & =-I^{1} \frac{d}{d t}(q(t) u(t))+I^{1} \frac{d}{d t}(h(t) f(u(t)))-q(0) u(0)+h(0) f(u(0)) \\
& =-q(t) u(t)+h(t) f(u(t))
\end{aligned}
$$

and then we get (1.1).

From (2.5) we have $u^{\prime}(0)=0$. Also by simple computation we can deduce from (2.1) that $\sum_{k=1}^{m} \xi_{k} u\left(a_{k}\right)=v \sum_{j=1}^{n} \eta_{j} u\left(b_{j}\right)$. Then problem (1.1)-(1.2) and Eq. (2.1) are equivalent.

Now from (2.5) using $\left(r_{3}\right)$, we have

$$
\begin{aligned}
u^{\prime \prime}(t) & \left.=\frac{d}{d t}\left[\frac{1}{p(t)} I^{\alpha}(-q(t) u(t))+h(t) f(u(t))\right)\right] \\
& =-\frac{p^{\prime}(t)}{p^{2}(t)} I^{\alpha}(-q(t) u(t)+h(t) f(u(t)))+\frac{1}{p(t)} I^{\alpha} \frac{d}{d t}(-q(t) u(t)
\end{aligned}
$$




$$
+h(t) f(u(t)))+\frac{1}{p(t)} \frac{t^{\alpha-1}}{\Gamma(\alpha)}(-q(0) u(0)+h(0) f(u(0)))
$$

and

$$
\begin{aligned}
\left|u^{\prime \prime}(t)\right| \leq & \frac{\left|p^{\prime}(t)\right|}{\left|p^{2}(t)\right|} \int_{0}^{t} \frac{(t-s)^{\alpha-1}}{\Gamma(\alpha)}(|q(s)||u(s)|+|h(s)||f(u(s))|) d s \\
& +\frac{1}{|p(t)|} \int_{0}^{t} \frac{(t-s)^{\alpha-1}}{\Gamma(\alpha)}\left(|q(s)|\left|u^{\prime}(s)\right|+\left|q^{\prime}(s)\right||u(s)|\right. \\
& \left.+|h(s)|\left|\frac{\partial f(u(s))}{\partial u}\right|\left|\frac{d u}{d s}\right|+\left|h^{\prime}(s)\right||f(u(s))|\right) d s \\
& +\frac{1}{|p(t)|} \frac{t^{\alpha-1}}{\Gamma(\alpha)}(|q(0)||u(0)|+|h(0)||f(u(0))|) .
\end{aligned}
$$

Therefore, we get

$$
\begin{aligned}
\int_{0}^{T}\left|u^{\prime \prime}(t)\right| d t \leq & \int_{0}^{T} \frac{\left|p^{\prime}(t)\right|}{\left|p^{2}(t)\right|} \int_{0}^{t} \frac{(t-s)^{\alpha-1}}{\Gamma(\alpha)}(|q(s)||u(s)|+|h(s)||f(u(s))|) d s d t \\
& +\int_{0}^{T} \frac{1}{|p(t)|} \int_{0}^{t} \frac{(t-s)^{\alpha-1}}{\Gamma(\alpha)}\left(|q(s)|\left|u^{\prime}(s)\right|+\left|q^{\prime}(s)\right||u(s)|\right. \\
& \left.+|h(s)|\left|\frac{\partial f(u(s))}{\partial u}\right|\left|u^{\prime}(s)\right|+\left|h^{\prime}(s)\right||f(u(s))|\right) d s d t \\
& +(|q(0)||u(0)|+|h(0)||f(u(0))|) \int_{0}^{T} \frac{1}{|p(t)|} \frac{t^{\alpha-1}}{\Gamma(\alpha)} d t \\
= & \int_{0}^{T}(|q(s)||u(s)|+|h(s)||f(u(s))|) d s \int_{s}^{T} \frac{\left|p^{\prime}(t)\right|}{\left|p^{2}(t)\right|} \frac{(t-s)^{\alpha-1}}{\Gamma(\alpha)} d t \\
& +\int_{0}^{T}\left(|q(s)|\left|u^{\prime}(s)\right|+\left|q^{\prime}(s)\right||u(s)|+|h(s)| \frac{\partial f(u(s))}{\partial u}|| u^{\prime}(s) \mid\right. \\
& \left.+\left|h^{\prime}(s)\right||f(u)|\right) d s \int_{s}^{T} \frac{1}{|p(t)|} \frac{(t-s)^{\alpha-1}}{\Gamma(\alpha)} d t \\
& +(|q(0) \| u(0)|+|h(0)||f(u(0))|) \int_{0}^{T} \frac{1}{|p(t)|} \frac{t^{\alpha-1}}{\Gamma(\alpha)} d t \\
\leq & (\|q\|\|u\|+\|h\|\|f\|) \frac{\left\|p^{\prime}\right\| T^{\alpha+1}}{p^{2} \Gamma(\alpha+1)}+\left(\|q\|\left\|u^{\prime}\right\| T+\left\|q^{\prime}\right\|_{L_{1}}\|u\|\right. \\
& \left.+\mathcal{K}\|h\|\left\|u^{\prime}\right\| T+\left\|h^{\prime}\right\|_{L_{1}} \mid f \|^{\prime}\right) \frac{T^{\alpha}}{p \Gamma(\alpha+1)} \\
& \frac{T^{\alpha}}{p \Gamma(\alpha+1)}(|q(0) \| u(0)|+|h(0)||f(u(0))|) .
\end{aligned}
$$

Then $u^{\prime \prime}(t) \in L_{1}(\mathcal{J}, \mathcal{R})$.

Define an operator $\mathcal{H}$ associated with the integral equation (2.1) as follows:

$$
\mathcal{H} u(t)=E \sum_{k=1}^{m} \xi_{k} \int_{0}^{a_{k}} \frac{1}{p(s)} I^{\alpha} q(s) u(s) d s-v E \sum_{j=1}^{n} \eta_{j} \int_{0}^{b_{j}} \frac{1}{p(s)} I^{\alpha} q(s) u(s) d s
$$




$$
\begin{aligned}
& -E \sum_{k=1}^{m} \xi_{k} \int_{0}^{a_{k}} \frac{1}{p(s)} I^{\alpha} h(s) f(u(s)) d s-\int_{0}^{t} \frac{1}{p(s)} I^{\alpha} q(s) u(s) d s \\
& +v E \sum_{j=1}^{n} \eta_{j} \int_{0}^{b_{j}} \frac{1}{p(s)} I^{\alpha} h(s) f(u(s)) d s+\int_{0}^{t} \frac{1}{p(s)} I^{\alpha} h(s) f(u(s)) d s .
\end{aligned}
$$

Theorem 2.2 Assume that the hypotheses $\left(D_{1}\right)-\left(D_{2}\right)$ hold, and the coefficients $\xi_{k}, \eta_{j}$ and $v \in \mathcal{R}$ with $\sum_{k=1}^{m} \xi_{k}-v \sum_{j=1}^{n} \eta_{j} \neq 0$. If

$$
\mathcal{G}=\frac{T^{\alpha+1}}{p \Gamma(\alpha+2)}\left[\left(|E|\left(\sum_{k=1}^{m}\left|\xi_{k}\right|+|\nu| \sum_{j=1}^{n}\left|\eta_{j}\right|\right)+1\right)(\|q\|+\mathcal{K}\|h\|)\right]<1,
$$

then Sturm-Liouville fractional differential equation (1.1) with the multi-point boundary condition (1.2) has a unique solution $u \in C^{1}(\mathcal{J}, \mathcal{R})$.

Proof Let $\sup _{t \in \mathcal{J}}|f(0)|=\mathcal{M}$; it follows from the Lipschitz condition that

$$
|f(u(t))|=|f(u)-f(0)+f(0)| \leq \mathcal{K}|u|+|f(0)| \leq \mathcal{K}\|u\|+\mathcal{M} .
$$

Firstly, we show that the operator $\mathcal{H}$ satisfies the relation $\mathcal{H} B_{r} \subset B_{r}$, where $B_{r}=\{u \in$ $C(\mathcal{J}, \mathcal{R}):\|u\| \leq r\}$ and

$$
r>\frac{\frac{\mathcal{M}\|h\| T^{\alpha+1}}{p \Gamma(\alpha+2)}\left[|E|\left(\sum_{k=1}^{m}\left|\xi_{k}\right|+|\nu| \sum_{j=1}^{n}\left|\eta_{j}\right|\right)+1\right]}{1-\mathcal{G}} .
$$

For $u \in B_{r}$, we have

$$
\begin{aligned}
& |\mathcal{H u}| \\
& \leq|E| \sum_{k=1}^{m}\left|\xi_{k}\right| \int_{0}^{a_{k}} \frac{1}{|p(s)|} I^{\alpha}|q(s)||u(s)| d s+|v||E| \sum_{j=1}^{n}\left|\eta_{j}\right| \int_{0}^{b_{j}} \frac{1}{|p(s)|} I^{\alpha}|q(s)||u(s)| d s \\
& +|E| \sum_{k=1}^{m}\left|\xi_{k}\right| \int_{0}^{a_{k}} \frac{1}{|p(s)|} I^{\alpha}|h(s)||f(u(s))| d s \\
& +|v||E| \sum_{j=1}^{n}\left|\eta_{j}\right| \int_{0}^{b_{j}} \frac{1}{|p(s)|} I^{\alpha}|h(s)||f(u(s))| d s \\
& +\int_{0}^{t} \frac{1}{|p(s)|} I^{\alpha}|q(s)||u(s)| d s+\int_{0}^{t} \frac{1}{|p(s)|} I^{\alpha}|h(s)||f(u(s))| d s \\
& \leq \frac{|E|\|q\|\|u\|}{p}\left[\sum_{k=1}^{m}\left|\xi_{k}\right| \int_{0}^{a_{k}} \int_{0}^{s} \frac{(s-\zeta)^{\alpha-1}}{\Gamma(\alpha)} d \zeta d s\right. \\
& \left.+|v| \sum_{j=1}^{n}\left|\eta_{j}\right| \int_{0}^{b_{j}} \int_{0}^{s} \frac{(s-\zeta)^{\alpha-1}}{\Gamma(\alpha)} d \zeta d s\right] \\
& +\frac{|E|\|h\|(\mathcal{K}\|u\|+\mathcal{M})}{p}\left[\sum_{k=1}^{m}\left|\xi_{k}\right| \int_{0}^{a_{k}} \int_{0}^{s} \frac{(s-\zeta)^{\alpha-1}}{\Gamma(\alpha)} d \zeta d s\right.
\end{aligned}
$$


El-Sayed and Gaafar Advances in Difference Equations

(2019) 2019:46

Page 7 of 17

$$
\begin{aligned}
& \left.+|v| \sum_{j=1}^{n}\left|\eta_{j}\right| \int_{0}^{b_{j}} \int_{0}^{s} \frac{(s-\zeta)^{\alpha-1}}{\Gamma(\alpha)} d \zeta d t\right]+\frac{\|q\|\|u\|}{p} \int_{0}^{t} \int_{0}^{s} \frac{(s-\zeta)^{\alpha-1}}{\Gamma(\alpha)} d \zeta d s \\
& +\frac{\|h\|(\mathcal{K}\|u\|+\mathcal{M})}{p} \int_{0}^{t} \int_{0}^{s} \frac{(s-\zeta)^{\alpha-1}}{\Gamma(\alpha)} d \zeta d s \\
& \leq \frac{|E|\|q\|\|u\|}{p}\left[\sum_{k=1}^{m}\left|\xi_{k}\right| \frac{a_{k}^{\alpha+1}}{\Gamma(\alpha+2)}+|\nu| \sum_{j=1}^{n}\left|\eta_{j}\right| \frac{b_{j}^{\alpha+1}}{\Gamma(\alpha+2)}\right] \\
& +\frac{|E|\|h\|(\mathcal{K}\|u\|+\mathcal{M})}{p}\left[\sum_{k=1}^{m}\left|\xi_{k}\right| \frac{a_{k}^{\alpha+1}}{\Gamma(\alpha+2)}+|\nu| \sum_{j=1}^{n}\left|\eta_{j}\right| \frac{b_{j}^{\alpha+1}}{\Gamma(\alpha+2)}\right] \\
& +\frac{\|q\|\|u\|}{p} \frac{T^{\alpha+1}}{\Gamma(\alpha+2)}+\frac{\|h\|(\mathcal{K}\|u\|+\mathcal{M})}{p} \frac{T^{\alpha+1}}{\Gamma(\alpha+2)} \\
& \leq \frac{T^{\alpha+1}}{p \Gamma(\alpha+2)}\left[\left(|E|\left(\sum_{k=1}^{m}\left|\xi_{k}\right|+|\nu| \sum_{j=1}^{n}\left|\eta_{j}\right|\right)+1\right)(\|q\|+\mathcal{K}\|h\|)\right]\|u\| \\
& +\frac{\mathcal{M}\|h\| T^{\alpha+1}}{p \Gamma(\alpha+2)}\left[|E|\left(\sum_{k=1}^{m}\left|\xi_{k}\right|+|\nu| \sum_{j=1}^{n}\left|\eta_{j}\right|\right)+1\right] \\
& \leq \frac{T^{\alpha+1}}{p \Gamma(\alpha+2)}\left[\left(|E|\left(\sum_{k=1}^{m}\left|\xi_{k}\right|+|\nu| \sum_{j=1}^{n}\left|\eta_{j}\right|\right)+1\right)(\|q\|+\mathcal{K}\|h\|)\right] r \\
& +\frac{\mathcal{M}\|h\| T^{\alpha+1}}{p \Gamma(\alpha+2)}\left[|E|\left(\sum_{k=1}^{m}\left|\xi_{k}\right|+|\nu| \sum_{j=1}^{n}\left|\eta_{j}\right|\right)+1\right] \leq r,
\end{aligned}
$$

which proves that $\mathcal{H} B_{r} \subset B_{r}$.

Now for $u, v \in C(\mathcal{J}, \mathcal{R})$ and $t \in \mathcal{J}$, we have

$$
\begin{aligned}
& |(\mathcal{H} u)(t)-(\mathcal{H} v)(t)| \\
& \leq|E| \sum_{k=1}^{m}\left|\xi_{k}\right| \int_{0}^{a_{k}} \frac{1}{|p(s)|} I^{\alpha}|q(s)||u(s)-v(s)| d s \\
& \quad+|v||E| \sum_{j=1}^{n}\left|\eta_{j}\right| \int_{0}^{b_{j}} \frac{1}{|p(s)|} I^{\alpha}|q(s)||u(s)-v(s)| d s \\
& \quad+|E| \sum_{k=1}^{m}\left|\xi_{k}\right| \int_{0}^{a_{k}} \frac{1}{|p(s)|} I^{\alpha}|h(s)||f(u(s))-f(v(s))| d s \\
& \quad+|v||E| \sum_{j=1}^{n}\left|\eta_{j}\right| \int_{0}^{b_{j}} \frac{1}{|p(s)|} I^{\alpha}|h(s)||f(u(s))-f(v(s))| d s \\
& \quad+\int_{0}^{t} \frac{1}{|p(s)|} I^{\alpha}|q(s)||u(s)-v(s)| d s+\int_{0}^{t} \frac{1}{|p(s)|} I^{\alpha}|h(s)| f(u(s))-f(v(s)) \mid d s \\
& \leq \\
& \quad \frac{|E||| q\||| u-v\|}{p}\left[\sum_{k=1}^{m}\left|\xi_{k}\right| \int_{0}^{a_{k}} \int_{0}^{s} \frac{(s-\zeta)^{\alpha-1}}{\Gamma(\alpha)} d \zeta d s\right. \\
& \left.\quad+|v| \sum_{j=1}^{n}\left|\eta_{j}\right| \int_{0}^{b_{j}} \int_{0}^{s} \frac{(s-\zeta)^{\alpha-1}}{\Gamma(\alpha)} d \zeta d s\right]
\end{aligned}
$$




$$
\begin{aligned}
& +\frac{\mathcal{K}|E|\|h\|\|u-v\|}{p}\left[\sum_{k=1}^{m}\left|\xi_{k}\right| \int_{0}^{a_{k}} \int_{0}^{s} \frac{(s-\zeta)^{\alpha-1}}{\Gamma(\alpha)} d \zeta d s\right. \\
& \left.+|v| \sum_{j=1}^{n}\left|\eta_{j}\right| \int_{0}^{b_{j}} \int_{0}^{s} \frac{(s-\zeta)^{\alpha-1}}{\Gamma(\alpha)} d \zeta d s\right] \\
& +\frac{\|q\|\|u-v\|}{p} \int_{0}^{t} \int_{0}^{s} \frac{(s-\zeta)^{\alpha-1}}{\Gamma(\alpha)} d \zeta d s+\frac{\mathcal{K}\|h\|\|u-v\|}{p} \int_{0}^{t} \int_{0}^{s} \frac{(s-\zeta)^{\alpha-1}}{\Gamma(\alpha)} d \zeta d s .
\end{aligned}
$$

By a similar calculation to the one above, we obtain

$$
\begin{aligned}
& |(\mathcal{H} u)(t)-(\mathcal{H} v)(t)| \\
& \quad \leq \frac{T^{\alpha+1}}{p \Gamma(\alpha+2)}\left[\left(|E|\left(\sum_{k=1}^{m}\left|\xi_{k}\right|+|v| \sum_{j=1}^{n}\left|\eta_{j}\right|\right)+1\right)(\|q\|+\mathcal{K}\|h\|)\right]\|u-v\| .
\end{aligned}
$$

Therefore, we get

$$
\begin{aligned}
& \|\mathcal{H} u-\mathcal{H} v\| \\
& \leq \frac{T^{\alpha+1}}{p \Gamma(\alpha+2)}\left[\left(|E|\left(\sum_{k=1}^{m}\left|\xi_{k}\right|+|v| \sum_{j=1}^{n}\left|\eta_{j}\right|\right)+1\right)(\|q\|+\mathcal{K}\|h\|)\right]\|u-v\| .
\end{aligned}
$$

It follows from (2.6) that $\mathcal{H}$ is a contraction mapping and by applying the Banach fixed point theorem problem $(1.1)-(1.2)$ has a unique solution $u \in C^{1}(\mathcal{J}, \mathcal{R})$.

Corollary 2.3 Let the assumptions $\left(D_{1}\right)-\left(D_{2}\right)$ be satisfied, and the coefficients $\xi_{k}, \eta_{j}$ and $v \in \mathcal{R}$ with $\sum_{k=1}^{m} \xi_{k}-v \sum_{j=1}^{n} \eta_{j} \neq 0$. If

$$
\frac{T^{2}}{2 p}\left[\left(|E|\left(\sum_{k=1}^{m}\left|\xi_{k}\right|+|v| \sum_{j=1}^{n}\left|\eta_{j}\right|\right)+1\right)(\|q\|+\mathcal{K}\|h\|)\right]<1,
$$

then the ordinary Sturm-Liouville differential equation

$$
\left(p(t) u^{\prime}(t)\right)^{\prime}+q(t) u(t)=h(t) f(u(t)), \quad t \in(0, T),
$$

under the conditions

$$
u^{\prime}(0)=0, \quad \sum_{k=1}^{m} \xi_{k} u\left(a_{k}\right)=v \sum_{j=1}^{n} \eta_{j} u\left(b_{j}\right)
$$

has a unique solution $u \in C^{1}(\mathcal{J}, \mathcal{R})$ if and only if $u$ solves the integral equation

$$
\begin{aligned}
u(t)= & E \sum_{k=1}^{m} \xi_{k} \int_{0}^{a_{k}} \frac{1}{p(s)} \int_{0}^{s} q(\zeta) u(\zeta) d \zeta d s-v E \sum_{j=1}^{n} \eta_{j} \int_{0}^{b_{j}} \frac{1}{p(s)} \int_{0}^{s} q(\zeta) u(\zeta) d \zeta d s \\
& -E \sum_{k=1}^{m} \xi_{k} \int_{0}^{a_{k}} \frac{1}{p(s)} \int_{0}^{s} h(\zeta) f(u(\zeta)) d \zeta d s
\end{aligned}
$$




$$
\begin{aligned}
& +v E \sum_{j=1}^{n} \eta_{j} \int_{0}^{b_{j}} \frac{1}{p(s)} \int_{0}^{s} h(\zeta) f(u(\zeta)) d \zeta d s \\
& -\int_{0}^{t} \frac{1}{p(s)} \int_{0}^{s} q(\zeta) u(\zeta) d \zeta d s+\int_{0}^{t} \frac{1}{p(s)} \int_{0}^{s} h(\zeta) f(u(\zeta)) d \zeta d s
\end{aligned}
$$

Proof Taking the limit as $\alpha \rightarrow 1$ in (1.1) and applying $\left(r_{3}\right)$ we get the ordinary SturmLiouville differential equation (2.9). Also from the continuity of the solution of (1.1)-(1.2) (cf. (2.1) and (2.6)) and applying $\left(r_{1}\right)$ we obtain (2.10) and (2.8), respectively.

Example Consider the Sturm-Liouville fractional differential equation

$$
{ }^{c} D^{1 / 2}\left(e^{t} u^{\prime}(t)\right)+\frac{\sqrt{t}}{100} u(t)=\frac{t}{20(t+1)} \tanh u(t), \quad t \in[0,1]
$$

under the conditions

$$
u^{\prime}(0)=0, \quad 4 u\left(\frac{1}{4}\right)-3 u\left(\frac{1}{3}\right)=\frac{1}{10}\left(7 u\left(\frac{2}{3}\right)-6 u\left(\frac{3}{4}\right)\right) .
$$

Here, $\alpha=\frac{1}{2}, p(t)=e^{t}, q(t)=\frac{\sqrt{t}}{100}, h(t)=\frac{t}{20(t+1)}, f(u(t))=\tanh u(t), \xi_{1}=4, \xi_{2}=-3, v=\frac{1}{10}$, $\eta_{1}=7, \eta_{2}=-6, T=1, p=1,\|q\|=\frac{1}{100},\|h\|=\frac{1}{40},\left|\frac{\partial f(u)}{\partial u}\right| \leq 1=\mathcal{K},|E|=\frac{10}{9}$, then $\mathcal{G}=$ $0.2691383936<1$.

Therefore by Theorem 2.2, the Sturm-Liouville fractional differential equation (2.11) under the conditions (2.12) has a unique continuous solution.

\section{Continuous dependence}

In this section we study the continuous dependence (on the coefficient $\xi_{k}$ and $\eta_{j}$ of the multi-point condition) of the solution of the Sturm-Liouville fractional differential equation (1.1) with the multi-point boundary condition (1.2).

Definition 3.1 The solution of the fractional Sturm-Liouville differential equation (1.1) is continuously dependent on the data $\xi_{k}$ and $\eta_{j}$ if for any $\epsilon>0$, there exist $\delta_{1}(\epsilon)$ and $\delta_{2}(\epsilon)$ such that, for any two solutions $u(t)$ and $\tilde{u}(t)$ of (1.1) with the initial data (1.2) and

$$
u^{\prime}(0)=0, \quad \sum_{k=1}^{m} \tilde{\xi}_{k} u\left(a_{k}\right)=v \sum_{j=1}^{n} \tilde{\eta}_{j} u\left(b_{j}\right)
$$

respectively, one has $\sum_{k=1}^{m}\left|\xi_{k}-\tilde{\xi}_{k}\right|<\delta_{1}$ and $\sum_{j=1}^{n}\left|\eta_{j}-\tilde{\eta}_{j}\right|<\delta_{2}$, then $\|u-\tilde{u}\|<\epsilon$ for all $t \in \mathcal{J}$.

Theorem 3.2 Let the assumptions of Theorem 2.2 be satisfied. Then the solution of SturmLiouville problem (1.1)-(1.2) is continuously dependent on the coefficients $\xi_{k}$ and $\eta_{j}$ of the multi-point boundary condition.

Proof Let $u(t)$ as defined in Eq. (2.1) be the solution of the multi-point problem (1.1)-(1.2) and

$$
\tilde{u}(t)=\tilde{E} \sum_{k=1}^{m} \tilde{\xi}_{k} \int_{0}^{a_{k}} \frac{1}{p(s)} I^{\alpha} q(s) \tilde{u}(s) d s-v \tilde{E} \sum_{j=1}^{n} \tilde{\eta}_{j} \int_{0}^{b_{j}} \frac{1}{p(s)} I^{\alpha} q(s) \tilde{u}(s) d s
$$




$$
\begin{aligned}
& -\tilde{E} \sum_{k=1}^{m} \tilde{\xi}_{k} \int_{0}^{a_{k}} \frac{1}{p(s)} I^{\alpha} h(s) f(\tilde{u}(s)) d s+v \tilde{E} \sum_{j=1}^{n} \tilde{\eta}_{j} \int_{0}^{b_{j}} \frac{1}{p(s)} I^{\alpha} h(s) f(\tilde{u}(s)) d s \\
& -\int_{0}^{t} \frac{1}{p(s)} I^{\alpha} q(s) \tilde{u}(s) d s+\int_{0}^{t} \frac{1}{p(s)} I^{\alpha} h(s) f(\tilde{u}(s)) d s
\end{aligned}
$$

be the solution of the nonlocal problem (1.1) and (3.1). Then

$$
\begin{aligned}
|u(t)-\tilde{u}(t)|= & \mid E \sum_{k=1}^{m} \xi_{k} \int_{0}^{a_{k}} \frac{1}{p(s)} I^{\alpha} q(s) u(s) d s-\tilde{E} \sum_{k=1}^{m} \tilde{\xi}_{k} \int_{0}^{a_{k}} \frac{1}{p(s)} I^{\alpha} q(s) \tilde{u}(s) d s \\
& -v E \sum_{j=1}^{n} \eta_{j} \int_{0}^{b_{j}} \frac{1}{p(s)} I^{\alpha} q(s) u(s) d s+v \tilde{E} \sum_{j=1}^{n} \tilde{\eta}_{j} \int_{0}^{b_{j}} \frac{1}{p(s)} I^{\alpha} q(s) \tilde{u}(s) d s \\
& -E \sum_{k=1}^{m} \xi_{k} \int_{0}^{a_{k}} \frac{1}{p(s)} I^{\alpha} h(s) f(u(s)) d s+\tilde{E} \sum_{k=1}^{m} \tilde{\xi}_{k} \int_{0}^{a_{k}} \frac{1}{p(s)} I^{\alpha} h(s) f(\tilde{u}(s)) d s \\
& +v E \sum_{j=1}^{n} \eta_{j} \int_{0}^{b_{j}} \frac{1}{p(s)} I^{\alpha} h(s) f(u(s)) d s-v \tilde{E} \sum_{j=1}^{n} \tilde{\eta}_{j} \int_{0}^{b_{j}} \frac{1}{p(s)} I^{\alpha} h(s) f(\tilde{u}(s)) d s \\
& -\int_{0}^{t} \frac{1}{p(s)} I^{\alpha} q(s) u(s) d s+\int_{0}^{t} \frac{1}{p(s)} I^{\alpha} q(s) \tilde{u}(s) d s \\
& +\int_{0}^{t} \frac{1}{p(s)} I^{\alpha} h(s) f(u(s)) d s-\int_{0}^{t} \frac{1}{p(s)} I^{\alpha} h(s) f(\tilde{u}(s)) d s \mid
\end{aligned}
$$

Now

$$
\begin{aligned}
&\left|E \sum_{k=1}^{m} \xi_{k} \int_{0}^{a_{k}} \frac{1}{p(s)} I^{\alpha} q(s) u(s) d s-\tilde{E} \sum_{k=1}^{m} \tilde{\xi}_{k} \int_{0}^{a_{k}} \frac{1}{p(s)} I^{\alpha} q(s) \tilde{u}(s) d s\right| \\
&=\mid E \sum_{k=1}^{m} \xi_{k} \int_{0}^{a_{k}} \frac{1}{p(s)} I^{\alpha} q(s) u(s) d s-\tilde{E} \sum_{k=1}^{m} \tilde{\xi}_{k} \int_{0}^{a_{k}} \frac{1}{p(s)} I^{\alpha} q(s) \tilde{u}(s) d s \\
& \quad-E \sum_{k=1}^{m} \xi_{k} \int_{0}^{a_{k}} \frac{1}{p(s)} I^{\alpha} q(s) \tilde{u}(s) d s+E \sum_{k=1}^{m} \xi_{k} \int_{0}^{a_{k}} \frac{1}{p(s)} I^{\alpha} q(s) \tilde{u}(s) d s \\
& \quad-\tilde{E} \sum_{k=1}^{m} \xi_{k} \int_{0}^{a_{k}} \frac{1}{p(s)} I^{\alpha} q(s) \tilde{u}(s) d s+\tilde{E} \sum_{k=1}^{m} \xi_{k} \int_{0}^{a_{k}} \frac{1}{p(s)} I^{\alpha} q(s) \tilde{u}(s) d s \mid \\
& \leq|E| \sum_{k=1}^{m}\left|\xi_{k}\right| \int_{0}^{a_{k}} \frac{1}{|p(s)|} I^{\alpha}|q(s)||u(s)-\tilde{u}(s)| d s \\
& \quad+|E-\tilde{E}| \sum_{k=1}^{m}\left|\xi_{k}\right| \int_{0}^{a_{k}} \frac{1}{|p(s)|} I^{\alpha}|q(s)||\tilde{u}(s)| d s \\
& \quad+|\tilde{E}| \sum_{k=1}^{m}\left|\xi_{k}-\tilde{\xi}_{k}\right| \int_{0}^{a_{k}} \frac{1}{|p(s)|} I^{\alpha}|q(s)||\tilde{u}(s)| d s \\
& \leq \frac{|E||| q\|\|\|u-\tilde{u}\| T^{\alpha+1}}{p \Gamma(\alpha+2)} \sum_{k=1}^{m}\left|\xi_{k}\right|+|\tilde{E}| \sum_{k=1}^{m}\left|\xi_{k}-\tilde{\xi}_{k}\right| \frac{\|q\|\|\tilde{u}\| T^{\alpha+1}}{p \Gamma(\alpha+2)}
\end{aligned}
$$




$$
\begin{aligned}
& +|E||\tilde{E}|\left(\sum_{k=1}^{m}\left|\xi_{k}-\tilde{\xi}_{k}\right|+|v| \sum_{j=1}^{n}\left|\eta_{j}-\tilde{\eta}_{j}\right|\right) \frac{\|q\|\|\tilde{u}\| T^{\alpha+1}}{p \Gamma(\alpha+2)} \sum_{k=1}^{m}\left|\xi_{k}\right| \\
\leq & \frac{|E|\|q\|\|u-\tilde{u}\| T^{\alpha+1}}{p \Gamma(\alpha+2)} \sum_{k=1}^{m}\left|\xi_{k}\right|+\delta_{1}|\tilde{E}| \frac{\|q\|\|\tilde{u}\| T^{\alpha+1}}{p \Gamma(\alpha+2)} \\
& +\left(\delta_{1}+|v| \delta_{2}\right)|E||\tilde{E}| \frac{\|q\|\|\tilde{u}\| T^{\alpha+1}}{p \Gamma(\alpha+2)} \sum_{k=1}^{m}\left|\xi_{k}\right| .
\end{aligned}
$$

Similar to (3.3) we can obtain

$$
\begin{aligned}
\mid-v E & \sum_{j=1}^{n} \eta_{j} \int_{0}^{b_{j}} \frac{1}{p(s)} I^{\alpha} q(s) u(s) d s+v \tilde{E} \sum_{j=1}^{n} \tilde{\eta}_{j} \int_{0}^{b_{j}} \frac{1}{p(s)} I^{\alpha} q(s) \tilde{u}(s) d s \mid \\
\leq & \frac{|v||E|\|q\|\|u-\tilde{u}\| T^{\alpha+1}}{p \Gamma(\alpha+2)} \sum_{j=1}^{n} \eta_{j}+|v| \delta_{2}|\tilde{E}| \frac{\|q\|\|\tilde{u}\| T^{\alpha+1}}{p \Gamma(\alpha+2)} \\
& +\left(\delta_{1}+|v| \delta_{2}\right)\left|v \left\||E \|| \tilde{E} \mid \frac{\|q\|\|\tilde{u}\| T^{\alpha+1}}{p \Gamma(\alpha+2)} \sum_{j=1}^{n} \eta_{j} .\right.\right.
\end{aligned}
$$

Also by a similar calculation to the one of (3.3) and by using (2.7), we obtain the following inequalities:

$$
\begin{aligned}
& \mid-E \sum_{k=1}^{m} \xi_{k} \int_{0}^{a_{k}} \frac{1}{p(s)} I^{\alpha} h(s) f(u(s)) d s+\tilde{E} \sum_{k=1}^{m} \tilde{\xi}_{k} \int_{0}^{a_{k}} \frac{1}{p(s)} I^{\alpha} h(s) f(\tilde{u}(s)) d s \mid \\
& \leq \frac{\mathcal{K}|E|\|h\|\|u-\tilde{u}\| T^{\alpha+1}}{p \Gamma(\alpha+2)} \sum_{k=1}^{m}\left|\xi_{k}\right|+\delta_{1}|\tilde{E}| \frac{\|h\|(\mathcal{K}\|\tilde{u}\|+\mathcal{M}) T^{\alpha+1}}{p \Gamma(\alpha+2)} \\
&+\left(\delta_{1}+|v| \delta_{2}\right)|E||\tilde{E}| \frac{\|h\|(\mathcal{K}\|\tilde{u}\|+\mathcal{M}) T^{\alpha+1}}{p \Gamma(\alpha+2)} \sum_{k=1}^{m}\left|\xi_{k}\right|, \\
&\left|v E \sum_{j=1}^{n} \eta_{j} \int_{0}^{b_{j}} \frac{1}{p(s)} I^{\alpha} h(s) f(u(s)) d s-v \tilde{E} \sum_{j=1}^{n} \tilde{\eta}_{j} \int_{0}^{b_{j}} \frac{1}{p(s)} I^{\alpha} h(s) f(\tilde{u}(s)) d s\right| \\
& \leq \frac{\mathcal{K}\left|v\||E|\| h\|\| u-\tilde{u} \| T^{\alpha+1} \sum_{j=1}^{n}\right| \eta_{j}\left|+\delta_{2}\right| v|| \tilde{E} \mid \frac{\|h\|(\mathcal{K}\|\tilde{u}\|+\mathcal{M}) T^{\alpha+1}}{p \Gamma(\alpha+2)}}{p \Gamma(\alpha+2)} \\
& \quad+\left(\delta_{1}+|v| \delta_{2}\right)|v\|E\| \tilde{E}| \frac{\|h\|(\mathcal{K}\|\tilde{u}\|+\mathcal{M}) T^{\alpha+1}}{p \Gamma(\alpha+2)} \sum_{j=1}^{n}\left|\eta_{j}\right| .
\end{aligned}
$$

Also it is easy to obtain

$$
\begin{aligned}
\mid-\int_{0}^{t} & \frac{1}{p(s)} I^{\alpha} q(s) u(s) d s+\int_{0}^{t} \frac{1}{p(s)} I^{\alpha} q(s) \tilde{u}(s) d s \\
& \quad+\int_{0}^{t} \frac{1}{p(s)} I^{\alpha} h(s) f(u(s)) d s-\int_{0}^{t} \frac{1}{p(s)} I^{\alpha} h(s) f(\tilde{u}(s)) d s \mid \\
\leq & \frac{\|q\|\|u-\tilde{u}\| T^{\alpha+1}}{p \Gamma(\alpha+2)}+\frac{\mathcal{K}\|h\|\|u-\tilde{u}\| T^{\alpha+1}}{p \Gamma(\alpha+2)} .
\end{aligned}
$$


With the final five inequalities, we have after reducing similar terms in Eq. (3.2):

$$
\begin{aligned}
\mid u(t) & -\tilde{u}(t) \mid \\
\leq & \frac{|E|\|q\|\|u-\tilde{u}\| T^{\alpha+1}}{p \Gamma(\alpha+2)}\left(\sum_{k=1}^{m}\left|\xi_{k}\right|+|v| \sum_{j=1}^{n}\left|\eta_{j}\right|\right)+\left(\delta_{1}+|v| \delta_{2}\right)|\tilde{E}| \frac{\|q\|\|\tilde{u}\| T^{\alpha+1}}{p \Gamma(\alpha+2)} \\
& +\left(\delta_{1}+|v| \delta_{2}\right)|E||\tilde{E}| \frac{\|q\|\|\tilde{u}\| T^{\alpha+1}}{p \Gamma(\alpha+2)}\left(\sum_{k=1}^{m}\left|\xi_{k}\right|+|v| \sum_{j=1}^{n}\left|\eta_{j}\right|\right) \\
& +\frac{\mathcal{K}|E|\|h\|\|u-\tilde{u}\| T^{\alpha+1}}{p \Gamma(\alpha+2)}\left(\sum_{k=1}^{m}\left|\xi_{k}\right|+|v| \sum_{j=1}^{n}\left|\eta_{j}\right|\right) \\
& +\left(\delta_{1}+|v| \delta_{2}\right)|\tilde{E}| \frac{\|h\|(\mathcal{K}\|\tilde{u}\|+\mathcal{M}) T^{\alpha+1}}{p \Gamma(\alpha+2)} \\
& +\left(\delta_{1}+|v| \delta_{2}\right)|E||\tilde{E}| \frac{\|h\|(\mathcal{K}\|\tilde{u}\|+\mathcal{M}) T^{\alpha+1}}{p \Gamma(\alpha+2)} \sum_{k=1}^{m}\left(\left|\xi_{k}\right|+|v| \sum_{j=1}^{n}\left|\eta_{j}\right|\right) \\
& +\frac{\|q\|\|u-\tilde{u}\| T^{\alpha+1}}{p \Gamma(\alpha+2)}+\frac{\mathcal{K}\|h\|\|u-\tilde{u}\| T^{\alpha+1}}{p \Gamma(\alpha+2)}
\end{aligned}
$$

and we get

$$
\begin{aligned}
\|u-\tilde{u}\| \leq & \frac{T^{\alpha+1}}{p \Gamma(\alpha+2)}\left[\left(|E|\left(\sum_{k=1}^{m}\left|\xi_{k}\right|+|\nu| \sum_{j=1}^{n}\left|\eta_{j}\right|\right)+1\right)(\|q\|+\mathcal{K}\|h\|)\right]\|u-\tilde{u}\| \\
& +\left(\delta_{1}+|\nu| \delta_{2}\right)|\tilde{E}| \frac{[\|q\|\|\tilde{u}\|+\|h\|(\mathcal{K}\|\tilde{u}\|+\mathcal{M})] T^{\alpha+1}}{p \Gamma(\alpha+2)} \\
& \times\left(|E|\left(\sum_{k=1}^{m}\left|\xi_{k}\right|+|\nu| \sum_{j=1}^{n}\left|\eta_{j}\right|\right)+1\right)
\end{aligned}
$$

then we have

$$
\begin{aligned}
(1-\mathcal{G})\|u-\tilde{u}\| \leq & \left(\delta_{1}+|v| \delta_{2}\right)|\tilde{E}| \frac{[\|q\|\|\tilde{u}\|+\|h\|(\mathcal{K}\|\tilde{u}\|+\mathcal{M})] T^{\alpha+1}}{p \Gamma(\alpha+2)} \\
& \times\left(|E|\left(\sum_{k=1}^{m}\left|\xi_{k}\right|+|v| \sum_{j=1}^{n}\left|\eta_{j}\right|\right)+1\right) .
\end{aligned}
$$

Therefore for $\sum_{k=1}^{m}\left|\xi_{k}-\tilde{\xi}_{k}\right|<\delta_{1}$ and $\sum_{j=1}^{n}\left|\eta_{j}-\tilde{\eta}_{j}\right|<\delta_{2}$, we can find

$$
\begin{aligned}
\|u-\tilde{u}\|<\epsilon= & (1-\mathcal{G})^{-1}\left(\delta_{1}+|\nu| \delta_{2}\right)|\tilde{E}| \frac{[\|q\|\|\tilde{u}\|+\|h\|(\mathcal{K}\|\tilde{u}\|+\mathcal{M})] T^{\alpha+1}}{p \Gamma(\alpha+2)} \\
& \times\left(|E|\left(\sum_{k=1}^{m}\left|\xi_{k}\right|+|\nu| \sum_{j=1}^{n}\left|\eta_{j}\right|\right)+1\right)
\end{aligned}
$$

i.e. for every $\epsilon>0$, there exist $\delta_{1}(\epsilon), \delta_{2}(\epsilon)>0$ such that $\sum_{k=1}^{m}\left|\xi_{k}-\tilde{\xi}_{k}\right|<\delta_{1}$ and $\sum_{j=1}^{n}\left|\eta_{j}-\tilde{\eta}_{j}\right|<$ $\delta_{2}$, then $\|u-\tilde{u}\|<\epsilon$. 
This proves the continuous dependence of the solution of the Sturm-Liouville fractional differential equation (1.1) with the multi-point boundary condition (1.2) on the coefficient $\xi_{k}$ and $\eta_{j}$ of the multi-point condition.

\section{Integral boundary conditions}

Let $u \in C^{1}(\mathcal{J}, \mathcal{R})$ be a solution of the problem (1.1) with the multi-point BCs in (1.2). Then we have the following theorem.

Theorem 4.1 Assume that the hypotheses $\left(D_{1}\right)-\left(D_{2}\right)$ hold. If

$$
\frac{T^{\alpha+1}}{p \Gamma(\alpha+2)}\left[\left(\frac{\varpi(c)-\varpi(a)+|v|(\vartheta(e)-\vartheta(d))}{|\varpi(c)-\varpi(a)-v(\vartheta(e)-\vartheta(d))|}+1\right)(\|q\|+\mathcal{K}\|h\|)\right]<1,
$$

then there exists a unique solution $u \in C^{1}(\mathcal{J}, \mathcal{R})$ of the fractional differential SturmLiouville problem:

$$
\begin{aligned}
& { }^{c} D^{\alpha}\left(p(t) u^{\prime}(t)\right)+q(t) u(t)=h(t) f(u(t)), \quad t \in[0, T], \\
& u^{\prime}(0)=0, \quad \int_{a}^{c} u(\theta) d \varpi(\theta)=v \int_{d}^{e} u(\theta) d \vartheta(\theta), \quad 0 \leq a<c \leq d<e \leq T,
\end{aligned}
$$

and $u$ solves (4.2) if and only if $u$ solves the integral equation

$$
\begin{aligned}
u(t)= & \frac{1}{\varpi(c)-\varpi(a)-v(\vartheta(e)-\vartheta(d))}\left[\int_{a}^{c} \int_{0}^{\theta} \frac{1}{p(s)} I^{\alpha} q(s) u(s) d s d \varpi(\theta)\right. \\
& -v \int_{d}^{e} \int_{0}^{\theta} \frac{1}{p(s)} I^{\alpha} q(s) u(s) d s d \vartheta(\theta)-\int_{a}^{c} \int_{0}^{\theta} \frac{1}{p(s)} I^{\alpha} h(s) f(u(s)) d s d \varpi(\theta) \\
& \left.+v \int_{d}^{e} \int_{0}^{\theta} \frac{1}{p(s)} I^{\alpha} h(s) f(u(s)) d s d \vartheta(\theta)\right] \\
& -\int_{0}^{t} \frac{1}{p(s)} I^{\alpha} q(s) u(s) d s+\int_{0}^{t} \frac{1}{p(s)} I^{\alpha} h(s) f(u(s)) d s,
\end{aligned}
$$

provided that $\varpi(c)-\varpi(a) \neq v(\vartheta(e)-\vartheta(d)), \varpi(\theta)$ and $\vartheta(\theta)$ are increasing functions and the integrals are meant in the Riemann-Stieltjes sense for $0 \leq a<c \leq d<e \leq T$.

Proof Let $u \in C[0, T]$ be a solution of problem (1.1)-(1.2). Let $\xi_{k}=\varpi\left(t_{k}\right)-\varpi\left(t_{k-1}\right)>0$, $a_{k} \in\left(t_{k-1}, t_{k}\right), 0 \leq a=t_{0}<t_{1}<t_{2}<\cdots<t_{m}=c, \eta_{j}=\vartheta\left(\tau_{j}\right)-\vartheta\left(\tau_{j-1}\right), b_{j} \in\left(\tau_{j-1}, \tau_{j}\right)$ and $d=\tau_{0}<$ $\tau_{1}<\cdots<\tau_{n}=e \leq T$. Then the multi-point boundary condition in (1.2) will be

$$
\sum_{k=1}^{m}\left(\varpi\left(t_{k}\right)-\varpi\left(t_{k-1}\right)\right) u\left(a_{k}\right)=v \sum_{j=1}^{n}\left(\vartheta\left(\tau_{j}\right)-\vartheta\left(\tau_{j-1}\right)\right) u\left(b_{j}\right),
$$

from the continuity of solution $u$ of (1.1)-(1.2), we can obtain

$$
\lim _{m \rightarrow \infty} \sum_{k=1}^{m}\left(\varpi\left(t_{k}\right)-\varpi\left(t_{k-1}\right)\right) u\left(a_{k}\right)=v \lim _{n \rightarrow \infty} \sum_{j=1}^{n}\left(\vartheta\left(\tau_{j}\right)-\vartheta\left(\tau_{j-1}\right)\right) u\left(b_{j}\right)
$$


that is, the multi-point boundary condition (1.2) is transformed to the integral condition

$$
\int_{a}^{c} u(\theta) d \varpi(\theta)=v \int_{d}^{e} u(\theta) d \vartheta(\theta)
$$

Next, from the continuity of the solution $u$ (cf. (2.1)), we can get

$$
\begin{aligned}
u(t)= & \frac{1}{\sum_{k=1}^{\infty} \xi_{k}-v \sum_{j=1}^{\infty} \eta_{j}} \lim _{m \rightarrow \infty} \sum_{k=1}^{m}\left(\varpi\left(t_{k}\right)-\varpi\left(t_{k-1}\right)\right) \int_{0}^{a_{k}} \frac{1}{p(s)} I^{\alpha} q(s) u(s) d s \\
& -\frac{v}{\sum_{k=1}^{\infty} \xi_{k}-v \sum_{j=1}^{\infty} \eta_{j}} \lim _{n \rightarrow \infty} \sum_{j=1}^{n}\left(\vartheta\left(\tau_{j}\right)-\vartheta\left(\tau_{j-1}\right)\right) \int_{0}^{b_{j}} \frac{1}{p(s)} I^{\alpha} q(s) u(s) d s \\
& -\frac{1}{\sum_{k=1}^{\infty} \xi_{k}-v \sum_{j=1}^{\infty} \eta_{j}} \lim _{m \rightarrow \infty} \sum_{k=1}^{m}\left(\varpi\left(t_{k}\right)-\varpi\left(t_{k-1}\right)\right) \int_{0}^{a_{k}} \frac{1}{p(s)} I^{\alpha} h(s) f(u(s)) d s \\
& +\frac{v}{\sum_{k=1}^{\infty} \xi_{k}-v \sum_{j=1}^{\infty} \eta_{j}} \lim _{n \rightarrow \infty} \sum_{j=1}^{n}\left(\vartheta\left(\tau_{j}\right)-\vartheta\left(\tau_{j-1}\right)\right) \int_{0}^{b_{j}} \frac{1}{p(s)} I^{\alpha} h(s) f(u(s)) d s \\
& -\int_{0}^{t} \frac{1}{p(s)} I^{\alpha} q(s) u(s) d s+\int_{0}^{t} \frac{1}{p(s)} I^{\alpha} h(s) f(u(s)) d s,
\end{aligned}
$$

and we see that $u \in C^{1}(\mathcal{J}, \mathcal{R})$ solves problem (4.2) if and only if $u$ solves (4.3).

Finally, substituting by $\xi_{k}$ and $\eta_{j}$ in (2.6) we get (4.1).

Corollary 4.2 Assume that the hypotheses $\left(D_{1}\right)-\left(D_{2}\right)$ hold. If

$$
\frac{T^{\alpha+1}}{p \Gamma(\alpha+2)}\left[\left(\frac{(c-a)+|v|(e-d)}{|(c-a)-v(e-d)|}+1\right)(\|q\|+\mathcal{K}\|h\|)\right]<1
$$

then there exists a unique solution $u \in C^{1}(\mathcal{J}, \mathcal{R})$ of the Sturm-Liouville fractional differential problem:

$$
\begin{aligned}
& { }^{c} D^{\alpha}\left(p(t) u^{\prime}(t)\right)+q(t) u(t)=h(t) f(u(t)), \quad t \in[0, T], \\
& u^{\prime}(0)=0, \quad \int_{a}^{c} u(\theta) d \theta=v \int_{d}^{e} u(\theta) d \theta, \quad 0 \leq a<c \leq d<e \leq T,
\end{aligned}
$$

and solving (4.4) is equivalent to finding a solution $u \in C^{1}(\mathcal{J}, \mathcal{R})$ to the integral equation

$$
\begin{aligned}
u(t)= & \frac{1}{c-a-v(e-d)}\left[\int_{a}^{c} \int_{0}^{\theta} \frac{1}{p(s)} I^{\alpha} q(s) u(s) d s d \theta\right. \\
& -v \int_{d}^{e} \int_{0}^{\theta} \frac{1}{p(s)} I^{\alpha} q(s) u(s) d s d \theta-\int_{a}^{c} \int_{0}^{\theta} \frac{1}{p(s)} I^{\alpha} h(s) f(u(s)) d s d \theta \\
& \left.+v \int_{d}^{e} \int_{0}^{\theta} \frac{1}{p(s)} I^{\alpha} h(s) f(u(s)) d s d \theta\right] \\
& -\int_{0}^{t} \frac{1}{p(s)} I^{\alpha} q(s) u(s) d s+\int_{0}^{t} \frac{1}{p(s)} I^{\alpha} h(s) f(u(s)) d s,
\end{aligned}
$$

provided that $c-a \neq v(e-d)$. 
Corollary 4.3 Let the assumptions $\left(D_{1}\right)-\left(D_{2}\right)$ be satisfied. If

$$
\frac{T^{2}}{2 p}\left[\left(\frac{\varpi(c)-\varpi(a)+|v|(\vartheta(e)-\vartheta(d))}{|\varpi(c)-\varpi(a)-v(\vartheta(e)-\vartheta(d))|}+1\right)(\|q\|+\mathcal{K}\|h\|)\right]<1,
$$

then the ordinary Sturm-Liouville differential problem

$$
\begin{aligned}
& \left(p(t) u^{\prime}(t)\right)^{\prime}+q(t) u(t)=h(t) f(u(t)), \quad t \in[0, T], \\
& u^{\prime}(0)=0, \quad \int_{a}^{c} u(\theta) d \varpi(\theta)=v \int_{d}^{e} u(\theta) d \vartheta(\theta), \quad 0 \leq a<c \leq d<e \leq T,
\end{aligned}
$$

has a unique solution $u \in C^{1}(\mathcal{J}, \mathcal{R})$ and solving (4.5), (4.6) is equivalent to finding a solution $u$ to the integral equation

$$
\begin{aligned}
u(t)= & \frac{1}{\varpi(c)-\varpi(a)-v(\vartheta(e)-\vartheta(d))}\left[\int_{a}^{c} \int_{0}^{\theta} \frac{1}{p(s)} \int_{0}^{s} q(\zeta) u(\zeta) d \zeta d s d \varpi(\theta)\right. \\
& -v \int_{d}^{e} \int_{0}^{\theta} \frac{1}{p(s)} \int_{0}^{s} q(\zeta) u(\zeta) d \zeta d s d \vartheta(\theta) \\
& -\int_{a}^{c} \int_{0}^{\theta} \frac{1}{p(s)} \int_{0}^{s} h(\zeta) f(u(\zeta)) d \zeta d s d \varpi(\theta) \\
& \left.+v \int_{d}^{e} \int_{0}^{\theta} \frac{1}{p(s)} \int_{0}^{s} h(\zeta) f(u(\zeta)) d \zeta d s d \vartheta(\theta)\right] \\
& -\int_{0}^{t} \frac{1}{p(s)} \int_{0}^{s} q(\zeta) u(\zeta) d \zeta d s+\int_{0}^{t} \frac{1}{p(s)} \int_{0}^{s} h(\zeta) f(u(\zeta)) d \zeta d s,
\end{aligned}
$$

provided that $\varpi(c)-\varpi(a) \neq v(\vartheta(e)-\vartheta(d))$.

Proof Taking the limit as $\alpha \rightarrow 1$ for (4.1), (4.2) and (4.3) and applying $\left(r_{1}\right)$ and ( $\left.r_{3}\right)$ we get the result.

Example Consider the Sturm-Liouville fractional differential equation

$$
{ }^{c} D^{3 / 4}\left(24\left(t^{2}+1\right) u^{\prime}(t)\right)+\sqrt[3]{t} u(t)=\sin t \tan ^{-1} u(t), \quad t \in(0,1),
$$

under the conditions

$$
u^{\prime}(0)=0, \quad \int_{0}^{\frac{1}{2}} u(\theta) d\left(\theta^{2}+1\right)=\frac{1}{100} \int_{3 / 4}^{1} u(\theta) d\left(\theta^{3}+2\right) .
$$

Here, $\alpha=3 / 4, p(t)=24\left(t^{2}+1\right), q(t)=\sqrt[3]{t}, h(t)=\sin t, f(u(t))=\tan ^{-1} u(t), c=1 / 2, \varpi(1 / 2)=$ 1.25, $a=0, \varpi(0)=1, v=\frac{1}{100}, e=1, \vartheta(1)=3, d=3 / 4, \vartheta(3 / 4)=2.41875, T=1, p=24$, $\|q\|=1,\|h\|=1,\left|\frac{\partial f(u)}{\partial u}\right| \leq 1=\mathcal{K}, \varpi(c)-\varpi(a)-v(\vartheta(e)-\vartheta(d))=0.24421875 \neq 0$.

Then

$$
\begin{aligned}
& \frac{T^{\alpha+1}}{p \Gamma(\alpha+2)}\left[\left(\frac{\varpi(c)-\varpi(a)+|v|(\vartheta(e)-\vartheta(d))}{|\varpi(c)-\varpi(a)-v(\vartheta(e)-\vartheta(d))|}+1\right)(\|q\|+\mathcal{K}\|h\|)\right] \\
& \quad=0.0530467051<1 .
\end{aligned}
$$


Therefore, by Theorem 4.1, the Sturm-Liouville fractional differential equation (4.7) under the conditions (4.8) has a unique continuous solution.

\author{
Acknowledgements \\ The authors would like to appreciate the anonymous reviewer for careful reading and very useful comments.
}

Funding

Not applicable.

\title{
Abbreviations
}

Not applicable.

\section{Competing interests}

The authors declare that they have no competing interests.

\section{Consent for publication}

Both authors read and approved the final version of the manuscript.

\section{Authors' contributions}

All authors contributed equally and read and approved the final version of the manuscript.

\section{Author details}

${ }^{1}$ Department of Mathematics, Faculty of Science, Alexandria University, Alexandria, Egypt. ${ }^{2}$ Department of Mathematics, Faculty of Science, Damanhour University, Damanhour, Egypt.

\section{Publisher's Note}

Springer Nature remains neutral with regard to jurisdictional claims in published maps and institutional affiliations.

Received: 2 June 2018 Accepted: 16 January 2019 Published online: 01 February 2019

\section{References}

1. Al-Mdallal, Q.M.: An efficient method for solving fractional Sturm-Liouville problems. Chaos Solitons Fractals 40, 183-189 (2009)

2. Bensidhoum, F.-Z., Dib, H.: On some regular fractional Sturm-Liouville problems with generalized Dirichlet conditions. J. Integral Equ. Appl. 28(4), 459-480 (2016)

3. Cui, Y., Zou, Y.: Nontrivial solutions of singular superlinear m-point boundary value problems. Appl. Math. Comput. $187,1256-1264(2007)$

4. EL-Sayed, A.M.A., Bin-Taher, E.O.: A nonlocal for a multi-term fractional order differential equation. Int. J. Math. Anal. 5(29), 1445-1451 (2011)

5. EL-Sayed, A.M.A., Bin-Taher, E.O.: Positive solutions for a nonlocal multi-point boundary-value problem od fractional and second order. Electron. J. Differ. Equ. 2013(64), 1 (2013)

6. El-Shahed, M., Nieto, J.J.: Nontrivial solutions for a nonlinear multi-point boundary value problem of fractional order. Comput. Math. Appl. 59, 3438-3443 (2010)

7. Erturk, V.S.: Computing eigenelements of Sturm-Liouville problems of fractional order via fractional differential transform method. Math. Comput. Appl. 16, 712-720 (2011)

8. Guo, Y., Yu, C., Wang, J.: Existence of three positive solutions for m-point boundary value problems on infinite intervals. Nonlinear Anal. 71, 717-722 (2009)

9. Hassana, A.A.: Green's function solution of non-homogenous regular Sturm-Liouville problem. J. Appl. Comput. Math. 6, 3 (2017). https://doi.org/10.4172/2168-9679.1000362

10. Joannopoulos, J.D., Johnson, S.G., Winnn, J.N., Meade, R.D.: Photonic Crystals: Molding the Folw of Light, 2 nd edn. Princeton University Press, Princeton (2008)

11. Karaaslan, M.F.: Numerical solution of a nonlocal fractional boundary value problem by Hdg method. J. Eng. Technol. Appl. Sci. 2(3), 121-129 (2017)

12. Klimek, M., Argawal, O.P.: On a regular fractional Sturm-Liouville problem with derivatives of order in $(0,1)$. In: 13 th Int. Cont. Conf. (2012)

13. Li, Y., Sun, S., Han, Z., Lu, H.: The existence of positive solutions for boundary value problem of the fractional Sturm-Liouville functional differential equation. Abstr. Appl. Anal. 2013, Article ID 301560 (2013)

14. Lian, H., Ge, W.: Existence of positive solutions for Sturm-Liouville boundary value problems on the half-line. J. Math. Anal. Appl. 321, 781-792 (2006)

15. Liang, S., Zhanga, J., Wangc, Z.: The existence of multiple positive solutions for multi-point boundary value problems on the half-line. J. Comput. Appl. Math. 228, 10-19 (2009)

16. Liu, X., Jia, M., Niu, M., Xiang, X.: Multiple positive solutions for multi-point boundary value problem of fractional differential equation. J. Math. Res. Appl. 37(2), 223-232 (2017)

17. Liu, Y., He, T., Shi, H.: Three positive solutions of Sturm-Liouville boundary value problems for fractional differential equations. Differ. Equ. Appl. 5(1), 127-152 (2013)

18. Ma, R.: Positive solutions of a nonlinear m-point boundary value problem. Comput. Math. Appl. 42, 755-765 (2002)

19. Miller, K.S., Ross, B.: An Introduction to the Fractional Calculus and Fractional Differential Equations. Wiley, New York (1993) 
20. Muensawat, T., Ntouyas, S.K., Tariboon, J.: Systems of generalized Sturm-Liouville and Langevin fractional differential equations. Adv. Differ. Equ. 2017, 63 (2017). https://doi.org/10.1186/s13662-017-1114-5

21. Nyamoradi, N.: Existence of solutions for multi point boundary value problems for fractional differential equations. Arab J. Math. Sci. 18, 165-175 (2012)

22. Nyamoradi, N.: Positive solutions for multi-point boundary value problems for nonlinear fractional differential equations. J. Contemp. Math. Anal. 48(4), 145-157 (2013)

23. Nyamoradi, N., Baleanu, D., Agarwal, R.P.: On a multipoint boundary value problem for a fractional order differential inclusion on an infinite interval, Adv. Math. Phys., 2013, Article ID 823961, 9 pages (2013). https://doi.org/10.1155/2013/82396

24. Podlubny, l.: Fractional Differential Equations. Academic Press, San Diego (1999)

25. Teschl, G.: Mathematical Methods in Quantum Mechanics: With Applications to Schrödinger Operators. Graduate Studies in Mathematics, vol. 99. Am. Math. Soc., Providence (2009)

26. Xu, J., Abernathy, Z.: On the solvability of nonlinear Sturm-Liouville problems. J. Math. Anal. Appl. 387, 310-319 (2012)

27. Yang, Z: Positive solutions for singular Sturm-Liouville boundary value problems on the half line. Electron. J. Differ. Equ. $2010171,1-8(2010)$

28. Zhang, X., Zhong, Q.: Multiple positive solutions for nonlocal boundary value problems of singular fractional differential equations. Bound. Value Probl. 2016, 65 (2016). https://doi.org/10.1186/s13661-016-0572-0

29. Zhanga, X., Liu, L.: Nontrivial solutions for higher order multi-point boundary value problems. Comput. Math. Appl. $56,861-873(2008)$

30. Zhao, X., Ge, W.: Existence of at least three positive solutions for multi-point boundary value problem on infinite intervals with p-Laplacian operator. J. Appl. Math. Comput. 28, 391-403 (2008)

31. Zhong, W., Lin, W.: Nonlocal and multiple-point boundary value problem for fractional differential equations. Comput. Math. Appl. 59(3), 1345-1351 (2010)

\section{Submit your manuscript to a SpringerOpen ${ }^{\circ}$ journal and benefit from:}

- Convenient online submission

- Rigorous peer review

Open access: articles freely available online

- High visibility within the field

- Retaining the copyright to your article

Submit your next manuscript at $\gg$ springeropen.com 